\title{
Identification of Rotor Unbalance as Inverse Problem of Measurement
}

\author{
Yuri Menshikov \\ Faculty of Mechanics \& Mathematics, Dnepropetrovsk University, Dnepropetrovsk, Ukraine \\ Email: Menshikov2003@list.ru
}

Received October 29, 2013; revised November 29, 2013; accepted December 5, 2013

Copyright (C) 2013 Yuri Menshikov. This is an open access article distributed under the Creative Commons Attribution License, which permits unrestricted use, distribution, and reproduction in any medium, provided the original work is properly cited. In accordance of the Creative Commons Attribution License all Copyrights (C) 2013 are reserved for SCIRP and the owner of the intellectual property Yuri Menshikov. All Copyright (C) 2013 are guarded by law and by SCIRP as a guardian.

\begin{abstract}
In this paper, the problem of identification of the characteristics of the rotor unbalance on two supports is investigated as the inverse problem of measurement. The vibration of rotor supports in two mutually perpendicular directions used as the initial information. The inverse problem is considered, taking into account the error of the mathematical description of rotor-bearings system. To obtain estimates of real unbalance characteristics, the hypothesis as to the exact solutions is applied. The method of Tikhonov regularization is used to obtain stable results. Test calculations are given to illustrate the proposed approach.
\end{abstract}

Keywords: Mathematical Model; Unbalance Identification; Solution Estimation; Regularization; Numerical Test

\section{Introduction}

The constructional differences of rotors and their domains of exploitation led to the creation of special methods of balancing. In many cases, the only criterion of rotor balancing is the absence (or the permissible value) of dynamical responses of supports. The compensation of deflections on length of rotor is considered only as the means to arrive of minimum of main criterion. In other cases, the reach of minimum of its deflections or its bending moment is taken as the criterion of rotor balancing. Such difference in choice of criterion can explain that for each case the parameters that are the main for given type of rotors are chosen. The reactions of supports or corresponding vibrations are taken for criteria of rotors balancing in particular to turbine-generator-building and the rotor deflection axis in jet engine building [1,2].

The basis of the most existing methods of flexible rotors balancing is the measuring of rotor vibrations and its supports, namely measuring of deflection and phases of rotating rotor followed by the choice and putting of trial plummets according to the shape of normal mode of vibrations.

The motion of flexible rotor relative to the rotating together with its coordinate system (one of axis coincides with the geometric axis of rotor) is described by Fred- holm equation of the first kind. This equation is substituted for the matrix equation of form:

$$
\boldsymbol{y}=\omega^{2} A(\boldsymbol{y}+\boldsymbol{e})=\omega^{2} A \boldsymbol{y}+\omega^{2} A \boldsymbol{e},
$$

where $\boldsymbol{y}$ and $\boldsymbol{e}$ are vectors of dimension $n$; $A=\left\{a_{i k}\right\}$ is the quadratic matrix of dimension $n \times n$; $\omega$ is the frequency of rotating. The coefficients of influence $A=\left\{a_{i k}\right\}$ are defined experimentally for each plane of correction by trial starts.

Balancing plummets are calculated with the help of the initial values of vibrations $y_{i}$ from condition of remaining amplitudes minimum. The definition of balancing plummets is made by least squares method. The information about experimental complex values of influence coefficients that are obtained by different balancing is neutralized as a rule.

The main tendencies of balancing methods development are connected with ways of development of coefficient influence definition [2,3].

Besides, current passive methods do not give the complete information about the position of unbalance if the rotor has a large length along the axis of rotation.

The suggested algorithms of unbalance evaluation use the experimental data about vibrations (accelerations) of two rotors' support in two mutually perpendicular directions during the work and a few rotor rotations as the 
initial information. These algorithms do not demand special conditions of work or the installation of trial plummets.

\section{Problem Definition}

Let us consider a deformable rotor rotating on two non-rigid supports $[4,5]$. We introduce rectangular righthand coordinates system $O \xi \eta \varsigma$. The axis $O \varsigma$ coincides with axis of the rotating shaft of rotor. The axis $O \xi$ belongs to the plane of rotor in horizontal position. The axis $O \eta$ has vertical direction. We obtain equations of rotor motion in the following $[4,5]$ :

1) Weight's centers and stiffness's centers of crosssection of rotor coincide;

2) Eccentricity of rotor's disk is one-order infinitesimal with a displacement under vibrations.

The motion of rotor on two non-region supports is described by system of ordinary differential equations of $18^{\text {th }}$ order [4,5]. Unbalance of rotor is modeled by some external load (EL). The value of this EL and the place of its action is it necessary to find. It is assumed that the vibrations of rotor supports in two mutual perpendicular directions are obtained from experiment. Let us suppose that the functions $z_{1}(t), z_{2}(t), z_{3}(t)$ characterize the unbalance of rotor (EL)

$$
\begin{aligned}
& z_{1}(t)=m_{r} r \dot{\varphi}^{2} \sin (\vartheta+\varphi), z_{2}(t)=m_{r} r \dot{\varphi}^{2} \cos (\vartheta+\varphi), \\
& z_{3}(t)=h m_{r} r \dot{\varphi}^{2} \sin (\vartheta+\varphi) ;
\end{aligned}
$$

where $r$ is the radius of rotor, $m_{r}$ is the mass of unbalance reducing to a surface of rotor, $\dot{\varphi}$ is the angular velocity of rotation, $h$ is unbalance arm, $\vartheta$ is angular deviation of the factor of EL with respect to correction plane. If the unbalance is absent then the functions $z_{1}(t), z_{2}(t), z_{3}(t)$ will be equal to zero. We suppose that with the help of acceleration transducers the function have been recorded $\left(\ddot{\xi}_{A}(t), \ddot{\xi}_{B}(t)\right.$ are the acceleration of supports in horizontal direction, $\ddot{\eta}_{A}(t), \ddot{\eta}_{B}(t)$ are the acceleration of supports in vertical direction). As an example, we consider the equation for the unknown function $z_{1}(t)$ only.

Then the problem of unbalance measurement is reduced to the solution of integral equations of Volltera first kind

$$
\int_{0}^{t}(t-\tau) z_{1}(t) \mathrm{d} \tau=u_{1}(t), t \in[0, T]
$$

or

$$
A_{1} z_{1}=B_{1, p} \boldsymbol{x}_{\delta}=u_{1, \delta},
$$

where $A_{1}$ is a linear integral operator $\left(A_{1}: Z \rightarrow U\right)$, $z_{1}$ is the searched characteristic of EL, $B_{1, p}$ is a linear irreversible operator $\left(B_{1, p}: X \rightarrow U\right)$ depending on vector parameters of mathematical model (MM) of "rotor- supports" system $p=\left(p_{1}, p_{2}, \cdots, p_{n}\right)^{\mathrm{T}}\left((\cdot)^{\mathrm{T}}\right.$ is the sign of transposition); $\boldsymbol{x}_{\delta}$ is the vector-function of initial data. Subjective factors influence on the definition of parameters of system "rotor-supports" MM and therefore the parameters are supposed to have their values within certain limits: $p_{i}^{l} \leq p_{i} \leq p_{i}^{t}, 1 \leq i \leq n$. In this way the vector $p$ can be changed inside the known closed region $p \in D \subset R^{n}$.

The equations for required functions $z_{2}(t), z_{3}(t)$ will be similar to the Equation (1).

For a rotor on two supports for function $z_{1}(t)$, $z_{2}(t), z_{3}(t)$ the vector parameters $p \in D \subset R^{11}$ of $\mathrm{MM}$ has a kind

$$
p=\left(E, J, m, m_{A}, m_{B}, c_{A}^{\xi}, c_{B}^{\xi}, b_{A}^{\xi}, b_{B}^{\xi}, a, b\right)^{\mathrm{T}},
$$

where $E$ is module of Jung of rotor material, $m$ is the mass of rotor, $m_{A}$ is the mass of the $A$ support, $m_{B}$ is the mass of the $B$ support; $c_{A}^{\xi}, c_{B}^{\xi}$ are the stiffness of supports $A$ and $B$ with respect to the horizontal and vertical direction; $b_{A}^{\xi}, b_{B}^{\xi}$ are the coefficients of external friction; $a$ is the distance of gravity centre of rotor to the $A$ support, $a+b=l$ is the shaft length of rotor.

The vector function $\boldsymbol{x}_{\delta}$ is obtained using the experimental data (vibrations of supports) where the noise is present. Therefore it is convenient to think that each component of vector function $\boldsymbol{x}_{\delta}$ and function $u_{\delta}$ belongs to $L_{2}[0, T]$. Under this conditions the problem of equation (1) solution belongs to ill-posed problems if the searched functions $z_{1}(t)$ belong to $C[0, T]$ as the operator $A$ in (1) is completely continuous [6].

The value of function deviation $u_{1, \delta}$ from the exact function $u_{e x}$ is given (if exact operator $B_{1, e x}$ is linear):

$$
\left\|u_{1, \delta}-u_{1, e x}\right\|_{U}=\left\|B_{1, p} \boldsymbol{x}_{\delta}-B_{1, e x} \boldsymbol{x}_{e x}\right\|_{U} \leq \delta_{0},\left\|A_{1}-A_{e x}\right\| \leq h,
$$

where $\delta_{0}=\delta b_{0}+d\left\|\boldsymbol{x}_{\delta}\right\|_{X}$;

$b_{0}=\sup _{p \in D}\left\|B_{1, p}\right\|_{X \rightarrow U},\left\|B_{1, p}-B_{1, e x}\right\|_{X \rightarrow U} \leq d,\left\|\boldsymbol{x}_{\delta}-\boldsymbol{x}_{e x}\right\|_{X} \leq \delta$;

$\boldsymbol{x}_{e x}$ is the exact vector function of initial data; $B_{1, e x}$, $A_{1, e x}$ are the exact operators; $\delta, b_{0}, d, h$ are given values.

\section{Statements of Identification Problem as Inverse Problem of Measurement}

Let us consider the set of possible solutions of Equation (1) with account of whole error of initial data

$$
Q_{h, \delta_{0}}=\left\{z: z \in Z,\left\|A_{1} z-B_{1, p} \boldsymbol{x}_{\delta}\right\|_{U} \leq \delta_{0}+h\|z\|_{Z}\right\} .
$$

The set $Q_{h, \delta_{0}}$ is unbounded for any $\delta_{0}>0$ as this problem is ill-posed [6].

For definition of stable approximate solution is used the regularization method of Tikhonov [6]. This way is based on the search of following extreme problem solu- 
tion:

$$
\Omega\left[z_{0}\right]=\inf _{z \in Q_{h, \delta_{0}}} \Omega[z]
$$

where $\Omega[z]$ is the stabilizing functional which is defined on $Z_{1}$ ( $Z_{1}$ is the everywhere dense set into $Z$ ).

The functional $\Omega[z]$ is chosen as follows

$$
\Omega[z]=\|z\|_{W_{1}^{2}[0, T]}^{2}=\int_{0}^{T}\left(q_{0} z^{2}+q_{1} \dot{z}^{2}\right) \mathrm{d} t,
$$

where $q_{0} \geq 0, q_{1}>0$.

The choice such functional is explained by the following reasons:

- The solution $z_{0}$ of an extreme problem (2) least will deviate zero and to have least first derivative in root-mean-square sense;

- The solution $z_{0}$ will give an estimation from below of exact solution $z_{T}$ of Equation (1) $\Omega\left[z_{0}\right] \leq \Omega\left[z_{T}\right]$.

From the practical point of view the function $z_{0}$ gives a guaranteed estimation from below sizes of real of a rotor in sense of functional $\Omega[z]$. If $\Omega^{0} \leq \Omega\left[z_{0}\right]$ ( $\Omega^{0}$ there is known limiting an allowable size for the given type of rotor machine) then the rotor is working in emergency operation with guarantee.

If the inequality $\Omega\left[z_{0}\right]<\Omega^{0}$ is carried out, then no objective conclusions can be made. We will be named the solution of extreme problem (2) as estimation from below of real unbalance.

In work [7] regularizing algorithm was suggested for Equation (1) with approximate linear operator $\tilde{A}$ for Banach spaces $Z, U$, which based on the regularization method [6].

The solution of problem (1) is reduced to the solution of following extreme problem:

$$
\begin{aligned}
\inf _{z \in Z_{1}} M^{\alpha}\left[z, u_{1, \delta}, A_{1}\right] & =\inf _{z \in Z_{1}}\left\{\left\|A_{1} z-u_{1, \delta}\right\|_{U}^{2}+\alpha \Omega[z]\right\} \\
& =M^{\alpha}\left[z_{1, \alpha}, u_{1, \delta}, A_{1}\right]
\end{aligned},
$$

where $\Omega[z]$ is stabilizing functional for Equation (1) which defined on $Z_{1}$, the set $Z_{1}$ is everywhere dense into $Z$.

Regularization parameter $\alpha$ can be obtained from equation of general discrepancy:

$$
\left\|A_{1} z_{1, \alpha}-u_{1, \delta}\right\|_{U}^{2}=\left(\delta_{0}+h\left\|z_{1, \alpha}\right\|_{Z_{1}}\right)^{2}+\mu^{2}\left(u_{1, \delta}, A_{1}\right),
$$

where $\mu\left(u_{1, \delta}, A_{1}\right)$ is measure of discrepancy.

However at realization of such approach there are large difficulties at definition of size $d, h$ as the absolute exact operators $B_{1, e x}, A_{1, e x}$ are unknown and basically they cannot be constructed. However at realization of such approach there are large difficulties at definition of size $d, h$ as the absolute exact operators $B_{1, e x}$ is unknown and basically it cannot be constructed. Therefore size $d$ is determined with the large overestimate and in set $Q_{d, \delta}$ the "extraneous" functions get, that considerably reduces accuracy of the regularized solution.

So in this paper the estimation of inverse problem solution instead of solution of Equation (1) is suggested. The following hypothesis is assumed for this purpose $[8,9]$ : the such inequality is valid

$$
\Omega\left[z_{1, e x}\right] \geq \Omega\left[z_{1, \alpha}\right]
$$

for any approximate operators $A_{1}, B_{1, p}$ in Equation (1) which corresponding to adequate mathematical description of vibration process [10]; $z_{1, e x}$ is the solution of Equation (1) with exact right part $u_{1, e x}$ and exact operator $A_{1, e x}$. The exact operators $A_{1, e x}, B_{1, e x}$ can are nonlinear.

The inequality (5) is evident if the exact operators are linear one.

In the given work it is supposed, that all approximate operators $B_{1, p}$ in (1) have the same structures which depend from some vector parameters $p \in D$. In this case extreme problem (2) can be replaced by the following extreme problem [11]:

$$
\Omega\left[z^{*}\right]=\inf _{z \in Q_{\delta}^{*}} \Omega[z]=\inf _{p \in D} \inf _{z \in Q_{p, \delta}} \Omega[z],
$$

where $Q^{*}=\bigcup_{p \in D} Q_{p, \delta} \quad(\bigcup$ is the union).

Now in set of the possible solutions "the extraneous functions" have not got.

It is evident that $Q^{*} \subset Q_{h, \delta}$ for any $d>0, h>0, \delta>0$. Therefore the use of the set $Q^{*}$ instead of $Q_{h, \delta}$ allows obtaining the most "thin" solution.

From the practical point of view the solution $z^{*}$ of an extreme problem (6) has the same meaning, as well as $z_{0}$, but gives an exacter estimation from below. We will be named the solution of extreme problem (6) as estimation from below of real unbalance also. But the inequality $\Omega\left[z_{0}\right] \leq \Omega\left[z^{*}\right]$ is valid.

For the solution of an extreme problem (6) it is offered to use a method of a choice of the minimal special mathematical model of system "rotor-supports" [11,12]. It allows getting more exact estimation of exact solution.

For the realization of such approach it is necessary to choose within the vectors $p \in D$ some vector $p_{0} \in D$ such that

$$
\Omega\left[A_{1}^{-1} B_{1, p_{0}} \boldsymbol{x}_{\delta}\right] \leq \Omega\left[A_{1}^{-1} B_{1, p} \boldsymbol{x}_{\delta}\right]
$$

for all possible $\boldsymbol{x}_{\delta} \in X$ and all $p \in D$. The operator $B_{1, p}$ with parameter $p_{0} \in D$ will be called the minimal operator. Appropriate to this operator the model is named as the minimal MM [12,13].

If the minimal MM exists, then the extreme problem (6) can be replaced by an equivalent simpler extreme problem:

$$
\Omega\left[z^{*}\right]=\inf _{z \in Q_{p_{o}, \delta}} \Omega[z] .
$$

Let's consider the problem on existence of the mini- 
mal MM in a problem of unbalance identification.

From physical sense of a problem follows, that the vibrations of support of a rotor $\ddot{\xi}_{A}(t), \ddot{\xi}_{B}(t), \dot{\xi}_{A}(t), \dot{\xi}_{B}(t)$, $\xi_{A}(t), \xi_{B}(t)$ at constant speed of rotation of a rotor $\omega$ and at constant size of unbalance in time $t \in[0, T]$ are periodic functions with zero average for the minimal period $T_{1}=2 \pi / \omega$.

The function $z_{1}(t)$ on physical sense represents also periodic function of a type $A_{1} \sin \left(\omega t+\varphi_{1}\right)$. At $\omega=$ const and at constant size of unbalance between functions $z_{1}(t), \xi_{A}(t)$ and $\xi_{B}(t)$, also there is a connection $\xi_{A}(t)=\mu^{-1} \xi_{B}(t)$, where $\mu$-Const, $\mu>0$.

Then the identity is valid

$$
\begin{aligned}
& \alpha_{1} \dddot{\xi}_{A}(t)+\alpha_{2} \dddot{\xi}_{A}(t)+\alpha_{3} \ddot{\xi}_{A}(t) \\
& +\alpha_{4} \dot{\xi}_{A}(t)+\alpha_{5} \xi_{A}(t)=z_{1}(t)
\end{aligned},
$$

where $\alpha_{1}, \alpha_{2}, \alpha_{3}, \alpha_{4}, \alpha_{5}$ are the function from $T$ and $p$.

In this case function $\Omega[z(p)]=\Omega[p]$ at the fixed functions $\xi_{A}(t), \xi_{B}(t)$ is continuous on components of a vector $p$. Under the well-known theorem of Weierstrass the function $\Omega[p]$ reaches on the convex closed set $D$ of the greatest lower bound.

Let's calculate partial derivatives of function $\Omega[p]$ on parameters (in the dimensionless form):

$$
\begin{gathered}
\frac{\partial \Omega}{\partial m}=\left(\varphi_{1} \varphi_{2}+\varphi_{1} k_{11}\right) k_{12}+k_{13}\left(\varphi_{1} \varphi_{2}+\varphi_{3} k_{14}\right), \\
\frac{\partial \Omega}{\partial(E J)}=-\left[\varphi_{1} \varphi_{4}+\varphi_{3} k_{12}+k_{22}\left(\varphi_{3} k_{23}+\varphi_{1} \varphi_{4}\right)\right], \\
\frac{\partial \Omega}{\partial m_{A}}=\varphi_{1} \varphi_{5} k_{31}, \frac{\partial \Omega}{\partial m_{B}}=\varphi_{1} \varphi_{6} k_{41}, \frac{\partial \Omega}{\partial a}=\varphi_{1} \varphi_{7} k_{51}+\varphi_{3} k_{52}, \\
\frac{\partial \Omega}{\partial b}=\varphi_{1} \varphi_{8} k_{61}+k_{62} \varphi_{3}+\left(\varphi_{3} k_{63}+\varphi_{1} k_{64}\right), \frac{\partial \Omega}{\partial b_{A}^{\xi}}=\varphi_{3} \varphi_{9} k_{71}, \\
\frac{\partial \Omega}{\partial b_{B}^{\xi}}=\varphi_{3} \varphi_{10} k_{81}, \frac{\partial \Omega}{\partial c_{A}^{\xi}}=\varphi_{1} \varphi_{11} k_{91}, \frac{\partial \Omega}{\partial c_{B}^{\xi}}=\varphi_{1} \varphi_{12} k_{92} ;
\end{gathered}
$$

where $k_{i k}$ are positive constants.

The signs of the partial derivatives are determined by signs and sizes of functions $\varphi_{1}, \varphi_{2}, \cdots, \varphi_{12}$, which depend on angular speed of rotation of a rotor $\omega$, parameters $p$ of MM and size of $T: \varphi_{k}=\varphi_{k}(\omega, p, T), k=\overline{1,12}$.

The functions $\varphi_{k}(k=1,12)$ from variable $z=\omega^{2} T^{2}$ are either square-law or linear. Therefore their signs are enough easily determined.

At rather small $T$ the minimal operator for (1) exists and associated the corner point of $D$.

In a problem of modeling of fluctuations in ventilator of the furnace the parameters of MM were the following [14]:

$$
m^{l}=0.84 \times 10^{4} \mathrm{~kg}, m^{t}=0.9 \times 10^{4} \mathrm{~kg},
$$

$$
\begin{gathered}
E^{l}=E^{t}=2 \times 10^{11} \mathrm{H} / \mathrm{m}^{2}, J^{l}=3 \times 10^{-3} \mathrm{~m}^{4}, \\
J^{t}=3.1 \times 10^{-3} \mathrm{~m}^{4}, \\
m_{A}^{l}=m_{B}^{l}=0.34 \times 10^{5} \mathrm{~kg}, m_{A}^{t}=m_{B}^{t}=0.35 \times 10^{5} \mathrm{~kg}, \\
c_{A}^{l}=c_{B}^{l}=0.65 \times 10^{9} \mathrm{H} / \mathrm{m}, c_{A}^{t}=c_{B}^{t}=0.7 \times 10^{9} \mathrm{H} / \mathrm{m}, \\
b_{A}^{l}=b_{B}^{l}=0, b_{A}^{t}=b_{B}^{t}=100 \mathrm{Hs} / \mathrm{m}^{2}, \\
a^{l}=5 \mathrm{~m}, a^{t}=5.1 \mathrm{~m}, b^{l}=3 \mathrm{~m}, b^{t}=3.1 \mathrm{~m} .
\end{gathered}
$$

At $T=0.1 \mathrm{~s}$ and $\omega=311 \mathrm{~s}^{-1}$ the value $\omega^{2} T^{2}$ has appeared less least roots of the equations $\varphi_{k}=0, k=\overline{1.12}$ (in view of allowable disorder of parameters). Therefore, in this example of function $\varphi_{k}$ have signs:

$$
\begin{gathered}
\varphi_{1}>0, \varphi_{3}<0, \varphi_{4}<0, \varphi_{5}<0, \varphi_{6}<0, \varphi_{7}<0, \varphi_{8}<0, \\
\varphi_{9}<0, \varphi_{10}<0, \varphi_{11}>0, \varphi_{12}>0 .
\end{gathered}
$$

Then, taking into account expressions (6), it is possible to conclude, that

$$
\begin{gathered}
\frac{\partial \Omega}{\partial m}<0, \frac{\partial \Omega}{\partial(E J)}>0, \frac{\partial \Omega}{\partial m_{A}}<0, \frac{\partial \Omega}{\partial m_{B}}<0, \frac{\partial \Omega}{\partial a}<0, \\
\frac{\partial \Omega}{\partial b}<0, \frac{\partial \Omega}{\partial b_{A}^{\xi}}>0, \frac{\partial \Omega}{\partial b_{B}^{\xi}}>0, \frac{\partial \Omega}{\partial c_{A}^{\xi}}>0, \frac{\partial \Omega}{\partial c_{A}^{\xi}}>0 .
\end{gathered}
$$

Hence, in a considered example the minimal model exists and corresponds to a vector

$$
p_{0}=\left(E^{l}, J^{l}, m^{t}, m_{A}^{t}, m_{B}^{t}, c_{A}^{l \xi}, c_{B}^{l \xi}, b_{A}^{l \xi}, b_{A}^{l \xi}, a^{t}, b^{t}\right)^{\mathrm{T}} .
$$

It is possibly that the size of $\Omega\left[z_{\delta, p}\right]$ for function $z_{\delta, p}$ from the some set $Q_{p, \delta}$ which satisfies to equality

$$
\Omega\left[z_{\delta, p}\right]=\inf _{z \in Q_{p, \delta}} \Omega[z],
$$

exceeds admissible size of $\Omega^{0}$. But the vector parameters $p$ of $\mathrm{MM}$ is given with error. To make this conclusion about a similar situation with a guarantee it is necessary to consider all possible sets $Q_{p, \delta}$, then in them to find all functions $\hat{z}_{p}$ which minimize $\Omega[z]$ on $Q_{p, \delta}$, further among them to find greatest in sense of functional value $\Omega\left[z_{\delta, p}\right]$. Thus we are getting to necessity of statement of the following problem:

$$
\Omega\left[\tilde{z}_{p}\right]=\sup _{p \in D} \inf _{z \in Q_{p, \delta}} \Omega[z] .
$$

It is obvious that $\Omega\left[z^{*}\right] \leq \Omega\left[\tilde{z}_{p}\right]$. If $\Omega^{0} \leq \Omega\left[\tilde{z}_{p}\right]$, then the machine probably works in emergency operation. If $\Omega\left[\tilde{z}_{p}\right] \leq \Omega^{0}$, then no certain conclusions can be made.

For the solution of an extreme problem (10) it is offered to use a method of a choice of the special maximal MM of system "rotor-supports".

It is possible to show that the solution of an extreme problem (10) under some conditions always exists. 
For the realization of such approach it is necessary to choose within the vectors $p \in D$ some vector $p^{1} \in D$ such that

$$
\Omega\left[A_{1}^{-1} B_{1, p} \boldsymbol{x}_{\delta}\right] \geq \Omega\left[A_{1}^{-1} B_{1, p} \boldsymbol{x}_{\delta}\right]
$$

for all possible $\boldsymbol{x}_{\delta} \in X$ and all $p \in D$. The operator $B_{1, p^{1}}$. with parameter $p^{1} \in D$ will be called the special maximal operator. Appropriate to this operator the model is named as the special maximal MM $[9,10]$.

If the special maximal MM exists then the solution of an extreme problem (10) will coincide with the solution of the following extreme problem:

$$
\Omega\left[\tilde{z}_{1, p}\right]=\inf _{z \in Q_{p^{1}, \delta}} \Omega[z] .
$$

For considered before an example the special maximal MM exists, unique and corresponds to a vector

$$
p^{1}=\left(E^{t}, J^{t}, m^{l}, m_{A}^{l}, m_{B}^{l}, c_{A}^{t \xi}, c_{B}^{t \xi}, b_{A}^{t \xi}, b_{A}^{t \xi}, a^{l}, b^{l}\right)^{\mathrm{T}} .
$$

If at a vector parameters $p$ is inexact the part of parameters is given only then a situation essentially to not change but only the vector parameters $p$ will have only smaller dimension. In a number of cases it is possible to carry out a choice of the special minimal or special maximal MM only in part of parameters of a vector $p$. And in this case it is possible to receive some prize in accuracy of the approximate estimation.

Let us consider the following statement of a problem of rotor unbalance identification: to find the function $z^{p l}$ among set of the possible solutions of the Equation (1) which would give the least maximal deviation from the experimentally measured vibrations of support of a rotor for all operators $B_{1, p}$. Such statement is reduced to the solution of the following extreme problem:

$$
\left\|A_{1} z^{p l}-B_{1, p} \boldsymbol{x}_{\delta}\right\|=\inf _{z_{p}} \sup _{B_{1, p}}\left\|A_{1} z_{p, \delta}-B_{1, p} \boldsymbol{x}_{\delta}\right\|_{U},
$$

where $z_{p, \delta}$ is the solution of extreme problem (11) on set $Q_{p, \delta}$.

As all operators $B_{1, p}$ it is possible to consider equivalent within the limits of the specified accuracy, it is possible to consider function $z^{p l}$ as the most probable solution of a problem of unbalance identification. The function $z^{p l}$ will be call the most plausible estimation of unbalance. The most probable estimation $z^{p l}$ will coincide with classical regularizing the solution of an inverse problem of unbalance identification of a rotor if there is the one operator $B_{1, p}$ only. The function $z^{p l}$ is the best approximation of real unbalance characteristics and also is steady to small deviations of the initial data.

Suggested algorithm can be used in case if the exact operator $B_{1, \text { ex }}$ does not belong to set of operators $B_{1, p}$ and the operator $A$ does not coincide with exact operator $A_{\text {ex }}$.

\section{Test Calculation}

For suggested algorithm examination of unbalance characteristics' evaluation, there was a calculated case when functions $\ddot{\xi}_{A}(t), \ddot{\xi}_{B}(t), \ddot{\eta}_{A}(t), \ddot{\eta}_{A}(t)$ are the results of mathematical simulation of rotor vibrations with given unbalance. The parameters of rotor unbalance were chosen as:

$$
m_{r}=0.5 \mathrm{~kg} \text { by } r=0.25 \mathrm{~m}, \mathrm{~h}=0.25 \mathrm{~m}, \vartheta=0.5 \mathrm{rad} \text {. }
$$

The values of initial data inaccuracy were chosen after filtering as the following:

$$
\begin{gathered}
\left\|\ddot{\xi}_{A}(t)-\ddot{\xi}_{A e x}(t)\right\|_{C} \leq \delta_{1}=0.08 \mathrm{~m} / \mathrm{s}^{2} \\
\left\|\ddot{\xi}_{B}(t)-\ddot{\xi}_{B e x}(t)\right\|_{C} \leq \delta_{2}=0.1 \mathrm{~m} / \mathrm{s}^{2} \\
\left\|\ddot{\eta}_{A}(t)-\ddot{\eta}_{A e x}(t)\right\|_{C} \leq \delta_{3}=0.1 \mathrm{~m} / \mathrm{s}^{2} \\
\left\|\ddot{\eta}_{B}(t)-\ddot{\eta}_{B e x}(t)\right\|_{C} \leq \delta_{4}=0.1 \mathrm{~m} / \mathrm{s}^{2}
\end{gathered}
$$

The whole inaccuracy of function $u_{d}(t)$ in Equation (1) is $\delta=0.266 \times 10^{4}$ by chosen inaccuracy of initial data. The discrepancy method defined the parameter of regularization $\alpha[7]$.

The functions $z_{1}(t), z_{2}(t), z_{3}(t)$ with parameters $m_{r}=0.02 \mathrm{~kg}, h=0.01 \mathrm{~m}, \vartheta=0.0 \mathrm{rad}$ are the results of identification as solution of extreme problem (2).

The results of identification with using the special minimal MM are followings:

$$
m_{r}=0.23 \mathrm{~kg}, h=0.12 \mathrm{~m}, \vartheta=0.45 \mathrm{rad} .
$$

The results of identification with using the special maximal $\mathrm{MM}$ are followings:

$$
m_{r}=0.37 \mathrm{~kg}, h=0.23 \mathrm{~m}, \vartheta=0.45 \mathrm{rad} .
$$

The solution of extreme problem (12) gives the results:

$$
m_{r}=0.41 \mathrm{~kg}, h=0.23 \mathrm{~m}, \vartheta=0.48 \mathrm{rad} .
$$

If the parameters of unbalance don't change during the 3 - 4 turns round, the axis of rotation is used for the parametric statement of problem. It permits to shorten the time of calculation of initial data about 10 times.

Efficiency of suggested algorithm was also shown on other tests $[1,3,12,15]$.

\section{REFERENCES}

[1] A. W. Lees and M. I. Friswell, "The Evaluation of Rotor Imbalance in Flexibly Mounted Machines," Journal of Sound and Vibration, Vol. 208, No. 5, 1997, pp. 671-683. http://dx.doi.org/10.1006/jsvi.1997.1260

[2] M. G. Smart, M. I. Friswell and A. W. Lees, "Estimating Turbogenerator Foundation Parameters: Model Selection and Regularization," Proceedings of the Royal Society of London A, Vol. 456, 2000, pp. 1583-1607. http://dx.doi.org/10.1098/rspa.2000.0577 
[3] M. S. Darlow, "Balancing of High-Speed Machinery: Theory, Methods and Experimental Results," Mechanical Systems and Signal Processing, Vol. 1, No. 1, 1982, pp. 105-134.

http://dx.doi.org/10.1016/0888-3270(87)90087-2

[4] Yu. L. Menshikov and N. V. Polyakov, "Operative Evaluation of Unbalance Characteristics of a Deformable Rotor," Proceedings of 8th International Symposium on Technical Diagnostics (IMEKO), Dresden, 23-25 September 1992, pp. 399-408.

[5] Yu. L. Menshikov and N. V. Polyakov, "The New Statement of Problem of Unbalance Identification," Proceedings of ICTAM 2004, Warsaw, 15-21 August 2004.

[6] A. N. Tikhonov and V. Y. Arsenin, "The Methods of Solution of the Incorrectly Formulated Problems," Science, Moscow, 1979.

[7] A. V. Goncharskij, A. C. Leonov and A. G. Yagola, "About One Regularized Algorithm for Ill-Posed Problems with Approximate Given Operator," Journal of Computational Mathematics and Mathematical Physics, Vol. 12, No. 6, 1972, pp. 1592-1594.

[8] Yu. L. Menshikov, "Useful Hypothesis in Inverse Problems of Interpretation," Proceedings of International Conference Inverse Problems and Applications (IPA2013), Linkoping University, Linköping, 2-6 April 2013.

[9] Yu. L. Menshikov, "Solution Estimations of Measure- ment's Inverse Problem," Proceedings of $4^{\text {th }}$ Inverse Problems, Design and Optimization Symposium (IPDO-2013), Book of Abstracts, Albi, 26-28 June 2013.

[10] Yu. L. Menshikov, "Synthesis of Adequate Mathematical Description as Solution of Special Inverse Problems," European Journal of Mathematical Sciences, Vol. 2, No 3, 2013, pp. 256-271.

[11] Yu. L. Menshikov, "The Reduction of Initial Date Inaccuracy in Ill-Posed Problems," Proceedings of 15th IMACS World Congress on Scientific Computation, Modelling and Applied Mathematics, Vol. VI, Berlin, 24-29 August 1997, pp. 577-582.

[12] Yu. L. Menshikov, "Identification of Optimal Mathematical Model of External Impacts," Proceedings of MATHMOD 03, Vienna, 5-7 February 2003.

[13] Yu. L. Menshikov, "Some New Statements of Inverse Problems of Recognition," 10th International Conference on Mathematical Methods in Electromagnetic Theory, Kharkov, 14-17 September 2004, pp. 464-466.

[14] Yu. L. Menshikov, "Recognition of Rotor Machines Unbalance," Differential Equations and Their Appendices in Physics. The Collection of the Proceedings, Dnepropetrovsk, 1988, pp. 44-56.

[15] Yu. L. Menshikov, "Identification of Rotor Unbalance in Minimax Statement," 13th International Congress on Sound and Vibration, Vienna, 2-6 July 2006, pp. 345-352. 